BMJ Open Ophthalmology

\title{
Orthokeratology with increased compression factor (OKIC): study design and preliminary results
}

\author{
Kin Wan (D), Jason Ki-kit Lau (D), Sin Wan Cheung, Pauline Cho
}

To cite: Wan K, Lau JK, Cheung SW, et al. Orthokeratology with increased compression factor (OKIC): study design and preliminary results. BMJ Open Ophthalmology 2020;5:e000345. doi:10.1136/ bmjophth-2019-000345

Received 22 May 2019 Revised 2 March 2020 Accepted 17 April 2020

\section{Check for updates}

C) Author(s) (or their employer(s)) 2020. Re-use permitted under CC BY-NC. No commercial re-use. See rights and permissions. Published by BMJ.

School of Optometry, The Hong Kong Polytechnic University, Hong Kong, China

Correspondence to KinWan; kkinwan@polyu.edu.hk

\section{ABSTRACT}

Objective To present the study design and the baseline data of a prospective cohort study investigating the safety, refractive correction and effectiveness of myopia control in subjects fitted with orthokeratology (ortho-k) lenses of different compression factors.

Methods and analysis This study is a 2-year Iongitudinal, double-masked, partially randomised study. Myopic children aged between 6 and 10 years are recruited and they may choose to participate in either the ortho-k or spectacle-wearing group. Subjects in the ortho-k group are randomly assigned to wear ortho-k lenses of either conventional compression factor (CCF, $0.75 \mathrm{D}$ ) or increased compression factor (ICF, $1.75 \mathrm{D}$ ). For the ortho-k subjects, the time and between-group effects within the first month of lens wear were analysed.

Results Sixty-nine ortho-k subjects (CCF: 34; ICF: 35) and 30 control subjects were recruited. There were no significant differences in baseline demographic data among the three groups of subjects $(p>0.19)$. At the 1-month visit, the first fit success rates were $97 \%$ and $100 \%$ in the CCF and ICF ortho-k group, respectively. A higher percentage of ICF subjects could achieve full correction (CCF: 88.2\%; ICF: 94.3\%). The change in axial length was significantly higher in the ICF group (CCF, $0.003 \mathrm{~mm}$; ICF, $-0.031 \mathrm{~mm})(\mathrm{p}<0.05)$. No significant between-group differences in daytime vision or in the coverage and depth of corneal staining between the two ortho-k groups ( $p>0.05$ ) were observed at any visit.

Conclusion ICF did not compromise the corneal integrity and the lens centration within the first month of lens wear. The preliminary performance of ortho-k lenses with ICF of $1.00 \mathrm{D}$ shows that it was safe to be used in the longer term for the investigation of myopia control.

Trial registration number NCT02643342.

\section{INTRODUCTION}

Compression factor (also known as Jessen factor) refers to the extra power, apart from the manifest refractive power of individuals, added on to the target of orthokeratology (ortho-k) lenses, in order to achieve an overcorrection $^{12}$ for counteracting the daytime regression after lens removal in the morning. ${ }^{3}$ The incorporated compression factor varies among different ortho-k lens manufacturers, but usually ranges from 0.50 to $0.75 \mathrm{D}$. Clinical studies have reported that the desired

\section{Key messages}

What is already known about this subject?

$>$ Orthokeratology (ortho-k) lenses with conventional compression factor (CCF) may not be adequate for achieving targeted refractive correction. The myopia control efficacy was relatively higher in the group of children with higher refractive errors.

\section{What are the new findings?}

Compared with CCF, in the increased compression factor (ICF), there was no difference in either the lens performance, in terms of centration, or the external ocular health. Although the desired over-correction could not be achieved in the ICF group, the axial length shortening was significantly higher than the CCF group at the 1-month visit, which may imply an increase in myopia control efficacy.

\section{How might these results change the focus of} research or clinical practice?

A long-term longitudinal study was warranted to confirm the effect of ICF, and this may lead to use of a new design in ortho-k lenses.

target reduction in refractive error may not be achieved with the conventional compression factor (CCF, $0.75 \mathrm{D}) .^{4-7}$ According to a retrospective analysis of the refraction at the second week of lens wear in 123 orthok-treated patients, ${ }^{8}$ an extra of $1.48 \mathrm{D}$ rather than $0.75 \mathrm{D}$ was required to obtain an overcorrection of $0.75 \mathrm{D}$.

Although evidence for ortho-k lenses in slowing down axial elongation has been well documented, ${ }^{9-15}$ the exact mechanism remains unknown. The myopic defocus to the retina induced by the cornea after ortho-k lenses wear has been proposed as the mechanism of slowing axial elongation. ${ }^{16}$ Therefore, the efficacy for myopia control of ortho-k lenses may be enhanced by increasing the steepening at the mid-peripheral cornea. The increased compression factor (ICF, $1.75 \mathrm{D}$ ) ortho-k can potentially achieve this purpose and induce a higher amount of peripheral retinal myopic defocus. Previous studies have shown promising results that greater corneal 
power changes were related to slower axial elongation, ${ }^{17-19}$ although Santodomingo-Rubido et al reported that there was no relationship between corneal power changes and either short-term or long-term axial elongation in ortho-k lens wearers. ${ }^{20}$ However, they did not account for the corneal power changes over the cornea across the entire treatment zone (including the steepened mid-peripheral annulus); considering only two points (paracentral $(3-5 \mathrm{~mm})$ and pericentral $(5-8 \mathrm{~mm})$ ) at the cornea, whose results might be significantly affected by lens decentration or corneal asymmetry. In view of the potentially enhanced myopia control efficacy for ICF ortho-k and to investigate the equivocal results on the association between corneal power changes and axial elongation, a long-term longitudinal study is warranted.

This 2-year longitudinal study is therefore conducted to determine the myopia control efficacy and long-term ocular responses of eyes fitted with ICF ortho-k lenses. In this report, the study design, and the lens performance of the ICF and CCF lenses, axial length (AL) and changes in central corneal thickness (CCT) in the ortho-k groups at the 1-month visit are presented.

\section{MATERIALS AND METHODS}

\section{Study design}

This is a partially randomised, double-blinded study to compare myopia control efficacy in subjects fitted with ICF or CCF ortho-k lenses and those wearing single-vision spectacles. The primary outcome is the axial elongation over a 2-year period.

Subjects are recruited via advertisements posted in local newspapers and through the internal email system of The Hong Kong Polytechnic University. Written informed consent is obtained from the parent/guardian before commencement of any procedures. Subjects may choose to participate in either the ortho-k or spectaclewearing group; however, ortho-k subjects are randomised to wear either CCF or ICF ortho-k lenses in both eyes according to a spreadsheet generator (Excel; Microsoft, Redmond, WA).

\section{Subjects}

Children of age 6 to not more than 12 years old, with myopia of $0.50 \mathrm{D}$ to $4.00 \mathrm{D}$ (inclusive) and astigmatism of less than $1.50 \mathrm{D}$, are recruited. Eligible subjects should be free of any ocular and systemic diseases and without any history of contact lens wear or myopia control treatment. The details of inclusion and exclusion criteria are listed in table 1.

Subjects are prescribed with ortho-k lenses (test groups) or single-vision spectacles (control group). Subjects in the ortho-k groups are randomly fitted with either CCF or ICF lenses in both eyes. They are masked to the compression factors of their lenses. They are required to follow the instructions of daily cleaning (Spray and Clean; Menicon, Japan), rinsing (Saline, Ophtecs, Japan), disinfection (MeniCare Plus; Menicon), weekly

\begin{tabular}{|c|c|}
\hline Inclusion criteria & Exclusion criteria \\
\hline $\begin{array}{l}6 \text { to } 11 \text { years old } \\
\text { (inclusive) }\end{array}$ & $\begin{array}{l}\text { Any type of strabismus or } \\
\text { amblyopia }\end{array}$ \\
\hline $\begin{array}{l}\text { Myopia: } 0.50-4.00 \mathrm{D} \text { in } \\
\text { both eyes }\end{array}$ & $\begin{array}{l}\text { Myopic control treatment } \\
\text { experience }\end{array}$ \\
\hline $\begin{array}{l}\text { Astigmatism: } \leq 1.50 \\
\text { D for with-the-rule } \\
\text { astigmatism (axes } \\
180 \pm 30 \text { ); } \leq 0.50 \mathrm{D} \text { for } \\
\text { other axes in both eyes }\end{array}$ & $\begin{array}{l}\text { Systemic or ocular } \\
\text { conditions which may affect } \\
\text { refractive development } \\
\text { (eg, Down's and Marfan's } \\
\text { syndrome, cataract and } \\
\text { ptosis) }\end{array}$ \\
\hline - Anisometropia: $\leq 1.00 \mathrm{D}$ & $\begin{array}{l}\text { Rigid contact lenses } \\
\text { experience }\end{array}$ \\
\hline $\begin{array}{l}\text { Symmetrical corneal } \\
\text { topography with corneal } \\
\text { toricity }<2.00 \mathrm{D} \text { in both } \\
\text { eyes }\end{array}$ & $\begin{array}{l}\text { Contraindications for } \\
\text { orthokeratology wear }\end{array}$ \\
\hline Agree to randomisation & $\begin{array}{l}\text { Poor compliance for lens } \\
\text { wear or follow-up }\end{array}$ \\
\hline
\end{tabular}

cleaning (Progent; Menicon), and monthly replacement of care products and lens cases.

\section{Interventions}

Ortho-k lenses

All ortho-k subjects are fitted with the Menicon Z Night ortho-k lenses (NKL Contactlenzen, The Netherlands) made of gas-permeable lens material (Menicon Z material, DK 163 ISO, central lens thickness $0.24 \mathrm{~mm}$ ) (table 2).

The initial lens parameters are determined using the computer software provided by the manufacturer (Easy Fit Software, V.2013; NKL Contactlenzen). The software uses the data obtained from the corneal topographer (Medmont E300 topographer; Medmont, Australia), subjective refraction and horizontal visible iris diameter to determine the lens parameters. Default parameters suggested by the software are ordered for the CCF group, but the compression factor is increased from $0.75 \mathrm{D}$ to $1.75 \mathrm{D}$ for ordering of lenses for the ICF group.

The target reduction in the two ortho-k groups is determined according to their baseline subjective refraction and remains unchanged throughout the whole study. A pair of single-vision over-spectacles, made of the same materials as those prescribed for the control subjects, are prescribed to correct the residual refractive errors for those with residual spherical equivalent refraction (SER) exceeding $0.50 \mathrm{D}$ in either eye or monocular aided vision worse than or equals to 0.18 (logMAR) after stabilisation in refractive correction of the ortho-k treatment. Spare lenses are ordered for the ortho-k subjects in case of any damage or loss. All lenses are replaced annually.

\section{Single-vision spectacles}

For the control subjects, single-vision spectacle lenses with spherical design and made of plastic material with 
Table 2 Lens details of Menicon Z Night lens

\begin{tabular}{|c|c|}
\hline Parameters & Description \\
\hline Material name & $\begin{array}{l}\text { Siloxanylstyrene } \\
\text { fluoromethacrylate (tisifilcon A) }\end{array}$ \\
\hline Dk (IOS) & $163 \times 10^{-11}$ \\
\hline Design & $\begin{array}{l}\text { Spherical/toric lens (parallel } \\
\text { reverse geometry) }\end{array}$ \\
\hline $\begin{array}{l}\text { Back optic zone radius } \\
(\mathrm{mm})\end{array}$ & $7.20-9.50$ (in 0.05 mm step) \\
\hline $\begin{array}{l}\text { Back optic zone diameter } \\
(\mathrm{mm})\end{array}$ & 6 \\
\hline Lens diameter (mm) & $10.20 / 10.60 / 11.00$ \\
\hline Reverse curve width & $1.2 \mathrm{~mm}$ for $10.6 \mathrm{~mm}$ lens \\
\hline Alignment curve width & $1.1 \mathrm{~mm}$ for $10.6 \mathrm{~mm}$ lens \\
\hline Tangential angle $\left({ }^{\circ}\right)$ & 50-65 (in $1^{\circ}$ step) \\
\hline Sagittal depth (mm) & 0.50-0.99 (in $0.01 \mathrm{~mm}$ step) \\
\hline Fenestration & $\begin{array}{l}\text { Three, located in the reverse } \\
\text { curve, } 120^{\circ} \text { apart }\end{array}$ \\
\hline Central thickness (mm) & 0.24 \\
\hline
\end{tabular}

refractive index of 1.60 are used (Hoya Lens, Japan). The prescription is updated when the monocular aided vision is 0.18 ( $\log$ MAR) or worse, or the change in SER is 0.50 $\mathrm{D}$ or greater.

\section{Examination schedules and procedures}

Cycloplegic examinations are performed at baseline and every 6 months on all subjects, after the commencement of the study (table 3 ).

These visits are all scheduled at about the same time of the day as the baseline visit to minimise the influence of diurnal variation on ocular parameters. Ortho-k subjects have to attend four extra follow-up visits: in the morning after the first overnight, first week, second week and 1 month after lens delivery. These visits aim to assess the lens performance and ocular health of the subjects. Unscheduled visits are provided if required.

Before the cycloplegic examination, two drops of $1 \%$ cyclopentolate applied $5 \mathrm{~min}$ apart are instilled. The pupillary response and the amplitude of accommodation are assessed after at least $30 \mathrm{~min}$. Cycloplegic measurements are performed on the eyes only when there is no pupillary response and the amplitude of accommodation is less than $2.00 \mathrm{D}$.

Objective refraction is measured with an open-field autorefractor (Shin-Nippon 5500K; Ajinomoto Trading, Japan). Five readings with less than $0.25 \mathrm{D}$ difference in both sphere and cylinder are recorded and averaged for analysis. Subjective refraction is conducted under standard clinical protocol with maximum plus for maximum visual acuity (VA). High-contrast $(>90 \%)$ VA is measured by using the Early Treatment Diabetic Retinopathy Study charts (Series 2000; Precision Vision, IL, USA). The Efron grading scale ${ }^{21}$ is used to grade ocular signs which were observed on biomicroscopy.

An optical biometer (Lenstar LS 900; Haag-Streit AG, Switzerland) is operated by the masked examiner for $\mathrm{AL}$ and CCT measurements. Five measurements with between-measurement difference of not more than $0.02 \mathrm{~mm}$ are recorded and averaged for analysis. The corneal profile is measured with a corneal topographer (Medmont E300 topographer; Medmont). Four measurements with each score higher or equal to 98 are recorded.

A Shack-Hartmann aberrometer (Complete Ophthalmic Analysis System (COAS); Wavefront Sciences, New Mexico, USA) is used to measure the ocular aberrations with different accommodation stimuli of $0 \mathrm{D}$ to $4 \mathrm{D}$ (1 D per step) before cycloplegia and $0 \mathrm{D}$

\begin{tabular}{llll}
\hline Table 3 Data collection schedule & & & \\
\hline Data collection visits & Baseline & 1-month visit & Every 6 months \\
\hline Refraction (subjective and objective) & & & \\
$\quad$ Pre-cycloplegic & $\mathrm{X}$ & $\mathrm{X}$ & $\mathrm{X}$ \\
\hline Post-cycloplegic & $\mathrm{X}$ & $\mathrm{X}$ \\
\hline High-contrast VA (habitual and best corrected) & & & $\mathrm{X}$ \\
$\quad$ Pre-cycloplegic & $\mathrm{X}$ & $\mathrm{X}$ & $\mathrm{X}$ \\
$\quad$ Post-cycloplegic & $\mathrm{X}$ & $\mathrm{X}$ & $\mathrm{X}$ \\
\hline Biomicroscopy & $\mathrm{X}$ & $\mathrm{X}$ & $\mathrm{X}$ \\
Corneal topography & $\mathrm{X}$ & $\mathrm{X}$ & $\mathrm{X}$ \\
Axial length & $\mathrm{X}$ & $\mathrm{X}$ \\
Ocular aberration & $\mathrm{X}$ & $\mathrm{X}$ \\
Corneal biomechanics & $\mathrm{X}$ & $\mathrm{X}$ \\
Anterior OCT & $\mathrm{X}$ & $\mathrm{X}$ \\
\hline Posterior OCT & $\mathrm{X}$ & & \\
\hline
\end{tabular}

*Only for subjects in both ortho-k groups.

OCT, optical coherence tomography; VA, visual acuity. 
after cycloplegia. A total of 125 measurements for each accommodation stimulus are averaged and used for analysis. The corneal biomechanical properties are measured with a dynamic bidirectional applanation device (Ocular response analyzer (ORA); Reichert, USA). Four measurements with waveform score higher or equal to 4.0 are recorded.

The anterior and posterior segment optical coherence tomography (OCT) images are captured with an anterior OCT (CASIA ss-1000; Tomey, Japan) and a posterior OCT (Spectralis OCT; Heidelberg Engineering, Germany), respectively. The anterior OCT images are captured under customised two-dimension and three-dimension scanning modes with auto alignment. The resolution for the two-dimension scanning mode is set to be 2048 A-scans with a scan range of $16 \mathrm{~mm}$ for vertical and horizontal meridians. The three-dimension scanning mode is set to be 16 radial scans across the $10 \mathrm{~mm}$ scan range across the cornea.

The posterior OCT images consist of six radial line scans of 30 degrees long and 30 degrees apart with enhanced depth imaging mode. The first image taken at the baseline visit is set as the reference. All the sequential images are scanned at the same location as the reference by using the manufacturer's 're-scan' function. Three OCT images of anterior and posterior segment are saved.

\section{Masking}

The primary outcome of the longitudinal study is the increase in AL. To eliminate any examiner bias, a masked examiner measures and records AL measurements.

\section{Sample size}

Based on the 2-year axial elongation reported in two previous studies $^{911}$ (ROMIO: $0.36 \pm 0.24 \mathrm{~mm}$, HMPRO: $0.19 \pm 0.21 \mathrm{~mm} ; 0.17 \mathrm{~mm}$ difference in elongation between ortho-k groups and SD of $0.21 \mathrm{~mm}$ ) and according to the equation by Sakpal, ${ }^{22}$ a minimal sample size of 24 subjects in each ortho-k group is required to give a power of $80 \%$ at a two-sided $5 \%$ significant level. Assuming 30\% dropouts, at least 34 subjects in each ortho-k group are recruited.

\section{Statistical analysis}

Following determination of normality of all data, most parameters were normally distributed, except cycloplegic subjective astigmatism. The significance level was set at 0.05 . Only the data from right eyes are presented in this report. The differences in baseline characteristics between groups were analysed using one-way analysis of variance (ANOVA) or Kruskal-Wallis test, where appropriate. For the investigation of the time and between ortho-k group effects of parametric parameters, repeated measures ANOVA and post hoc tests with Bonferroni correction were used. For astigmatism, Friedman test and Mann-Whitney U test with Bonferroni correction were used, respectively. The refractive data were also transformed into Long's formalism (SCxA) and the time

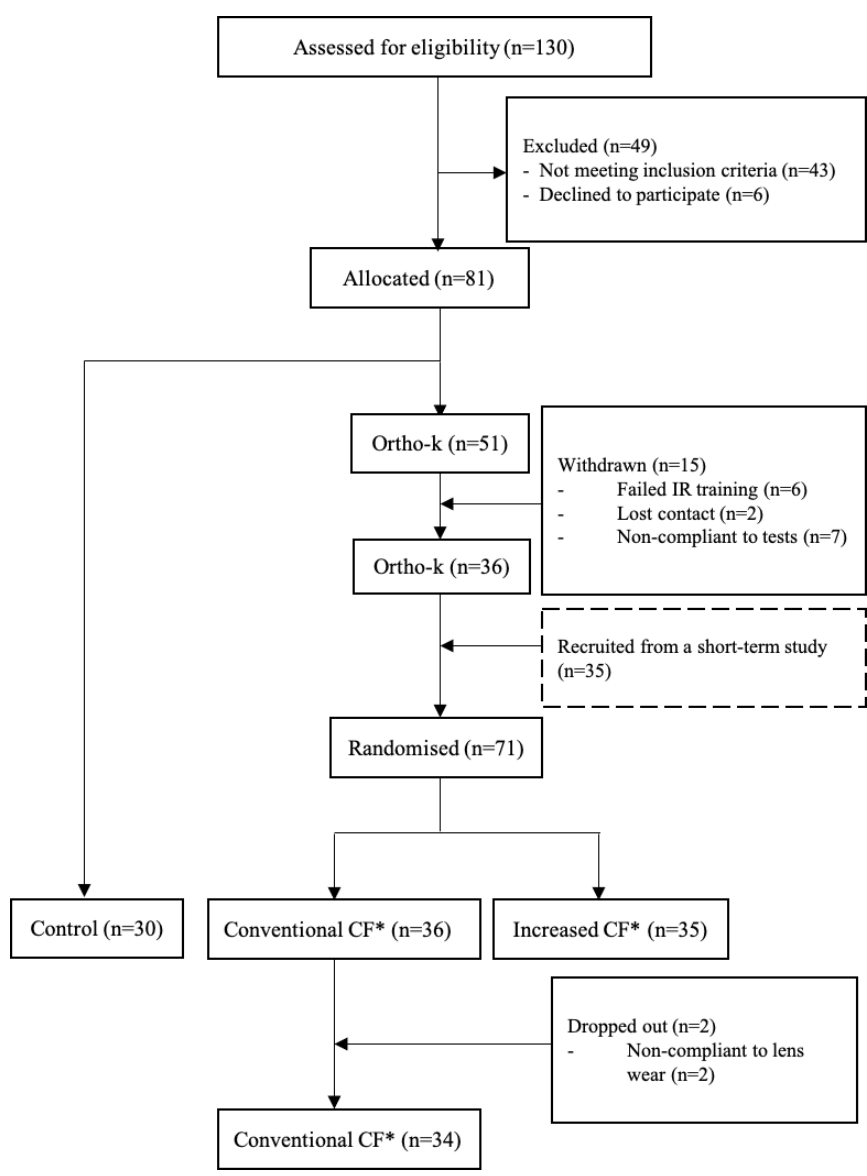

Figure 1 Flowchart of the conduct of the study. ${ }^{*} \mathrm{CF}$, compression factor.

effect was tested by using Hotelling's T-squared. ${ }^{23}$ To evaluate the difference in external ocular health between two ortho-k groups, Mann-Whitney U test was used for ordinal data.

\section{Patient and public involvement}

Patients were not invited to comment on the study design and were not consulted to develop patient relevant outcomes or interpret the results. Patients were not involved in writing or editing of this article.

\section{RESULTS}

Thirty-six and 35 subjects were fitted with CCF and ICF lenses, respectively, and 30 subjects were fitted with singlevision spectacles (figure 1). Two subjects in the CCF group were excluded before the 1-month visit because of non-compliance with lens wear. Thirty-four subjects in the CCF group and 35 subjects in the ICF group completed the 1-month visit. At 2-week visit, one CCF subject needed to be refitted because of lens decentration, yielding a first fit success rate of $97 \%(33 / 34)$ and $100 \%(35 / 35)$ for $\mathrm{CCF}$ and ICF groups, respectively.

Table 4 shows a summary of the baseline data for the control and two groups of ortho-k subjects who completed the 1-month visit. No significant differences in baseline age, pre-cycloplegic and post-cycloplegic subjective refraction, high-contrast unaided and best-corrected 
Table 4 Mean \pm SD (or median (range)) age and ocular parameters of the subjects at the commencement of the study

\begin{tabular}{lllll} 
& Control $(\mathbf{n}=\mathbf{3 0})$ & CCF $(\mathbf{n}=\mathbf{3 4})$ & ICF $(\mathbf{n}=\mathbf{3 5})$ & *P value \\
\hline Age (years) & $9.01 \pm 0.99$ & $9.03 \pm 1.03$ & $9.42 \pm 1.06$ & 0.19 \\
\hline Pre-cyclo S (D) & $-2.07 \pm 0.80$ & $-2.19 \pm 0.77$ & $-2.16 \pm 0.84$ & 0.97 \\
\hline Pre-cyclo C (D) & $-0.25(0.00$ to -1.50$)$ & $-0.25(0.00$ to -1.25$)$ & $-0.25(0.00$ to -1.50$)$ & $0.61 \dagger$ \\
\hline Pre-cyclo SCxA & $-2.16-0.25 \times 177$ & $-2.21-0.34 \times 001$ & $-2.20-0.31 \times 002$ & \\
\hline Post-cyclo S (D) & $-2.00 \pm 0.82$ & $-2.17 \pm 0.81$ & $-2.12 \pm 0.86$ & 0.95 \\
\hline Post-cyclo C (D) & $-0.50(0.00$ to -1.50$)$ & $-0.50(0.00$ to -1.50$)$ & $-0.25(0.00$ to -1.50$)$ & $0.67 \dagger$ \\
\hline Post-cylco SCxA & $-2.10-0.32 \times 180$ & $-2.12-0.38 \times 001$ & $-2.09-0.33 \times 179$ & \\
\hline Unaided logMAR VA & $0.65 \pm 0.25$ & $0.64 \pm 0.27$ & $0.67 \pm 0.30$ & 0.90 \\
\hline Best-corrected logMAR VA & $-0.02 \pm 0.06$ & $-0.02 \pm 0.06$ & $-0.02 \pm 0.06$ & 0.95 \\
\hline CCT $(\mu \mathrm{m})$ & $547 \pm 35$ & $545 \pm 30$ & $552 \pm 28$ & 0.64 \\
\hline Axial length $(\mathrm{mm})$ & $24.39 \pm 0.81$ & $24.36 \pm 0.66$ & $24.51 \pm 0.83$ & 0.47 \\
\hline
\end{tabular}

Values are presented as mean \pm SD or median (range).

*Probability values of one-way analysis of variance (ANOVA) for between-group difference.

†Probability value of Kruskal-Wallis test for between-group difference.

CCF, conventional compression factor; CCT, central corneal thickness; ICF, increased compression factor; Post-cyclo C, post-cycloplegic subjective astigmatism; Pre-cyclo C, pre-cycloplegic subjective astigmatism; Post-cyclo S, post-cycloplegic subjective sphere; Pre-cyclo S, pre-cycloplegic subjective sphere; SCxA, compound refractive number after Long's formalism; VA, visual acuity.

visual acuity, and AL were found among the three groups $(\mathrm{p}>0.05)$.

Ocular characteristics of the ortho-k subjects after 1 month of Iens wear

Changes in myopia for CCF and ICF groups are shown in figure 2. The refractive errors (SCxA) at 1-month visit were $0.12-0.20 \times 10$ and $0.42-0.13 \times 19$ for $\mathrm{CCF}$ and ICF, respectively. Myopia in the ICF group were significantly more positive than the CCF group from the 1-week visit onward $(p<0.05)$. No significant reduction in astigmatism was observed in either group of subjects $(p>0.05)$, and no significant differences were observed between the two groups at all visits $(p>0.05)$ during the first month of lens wear. Full correction is defined as residual myopia of less than 0.50 D. Four subjects in the CCF group (11.8\%)

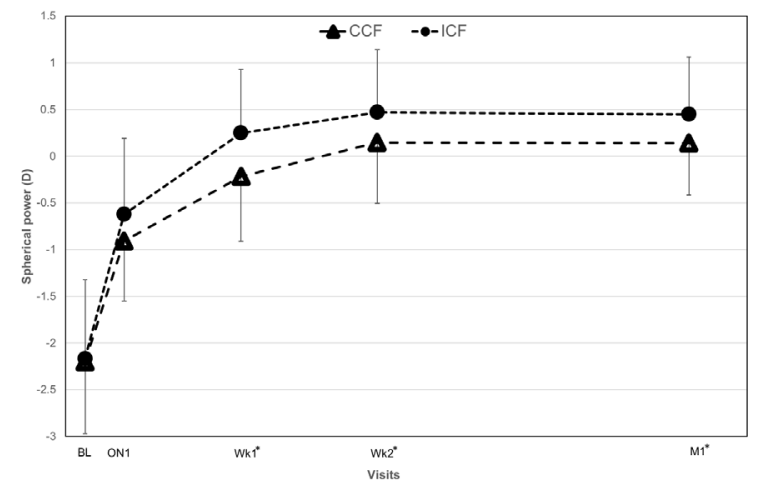

Figure 2 Changes in myopia in ortho-k subjects wearing lenses of conventional compression factor (CCF) and increased compression factor (ICF). Error bars indicate the $\mathrm{SD}$. The asterisk $\left(^{*}\right)$ denotes a significant difference in myopia between groups. BL, baseline; ON1, first overnight; Wk1, first week; Wk2, second week; M1, 1 month. and two subjects in the ICF group (5.7\%) did not achieve full correction at the 1-month visit. No significant difference in high-contrast unaided VA and best-corrected VA between the two ortho-k groups was observed at any visit $(\mathrm{p}<0.05)$.

The coverage and depth of corneal staining observed during the first month of lens wear were not more than grade 2 in all ortho-k subjects. Central corneal staining observed in both groups at different visits are presented in figure 3. There were no significant differences in either coverage or depth of corneal staining between the two groups ( $>0.05)$ at any visit. Corneal pigmented arc was observed in one subject in the CCF group at the 1-month visit and none in the ICF group. No infectious keratitis or any other serious ocular conditions occurred during the 1 month of lens wear.

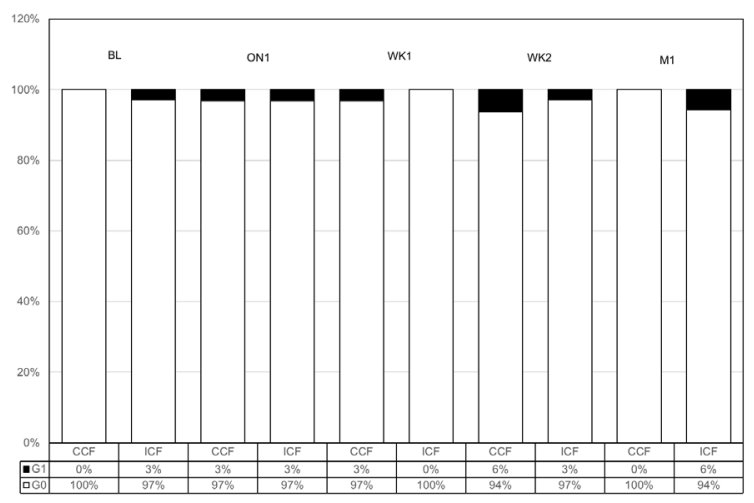

Figure 3 Severity (coverage) of central corneal staining in conventional compression factor (CCF) and increased compression factor (ICF) groups. BL, baseline; ON1, first overnight; WK1, first week; WK2, second week; M1, 1 month; G0-G1: grade 0 to grade 1. 
$\mathrm{AL}$ was reduced in the ICF group (mean (SD) change: $-0.031(0.060) \mathrm{mm} ; \mathrm{p}<0.05)$ but remained unchanged in the CCF group (mean (SD) change: $0.003(0.038) \mathrm{mm}$; $\mathrm{p}>0.05)$ at the 1-month visit. The between-group difference in AL change was significant $(p<0.05)$. CCT were reduced in both ortho-k groups $(\mathrm{p}<0.05)$ from baseline to the 1-month visit, although the between-group difference was not significant $(p>0.05)$. The mean (SD) changes in CCT were -7.03 (8.06) and -10.34 (5.97) $\mu \mathrm{m}$, respectively.

\section{DISCUSSION}

This report presents the study design and the preliminary findings of ortho-k subjects participating in a longitudinal study exploring the efficacy of myopia control using ortho-k with higher compression factor. Although an extra $1 \mathrm{D}$ target was added in the ICF group, there was only $0.30 \mathrm{D}$ difference in the manifest refractive sphere between ICF and CCF groups. The results were in agreement with an earlier short-term study. ${ }^{24}$ The undercorrection could be due to several factors. The first factor could be the time of data collection. The amount of manifest refraction could be dependent on the time of day when the measurement was made, the reported daytime regression by previous investigators was about $0.25 \mathrm{D}$ to $0.50 \mathrm{D}^{32526}$ or even less than $0.25 \mathrm{D}^{27}$; however, this factor should affect both the ortho-k groups and contribute minimally to the between-group difference. Although this factor could potentially affect the measured manifest refraction, subjects could not all be measured at the same time because of the limited resources. In this study, all measurements were performed during daytime and the diurnal variation was controlled by scheduling all the data collection visits at about the same time of day as the baseline visit. The second potential contributing factor could be the relatively short wearing period: the increased target may take a longer time to stabilise, although a previous study has shown that the manifest refractive power stabilised after 1 month of lens wear. ${ }^{27}$ Another explanation is that there may be a corneal mechanism, which is related to the corneal structural properties, ${ }^{28}$ that could limit the amount of corneal changes, hence affecting the manifest refractive power changes.

Peripheral corneal steepening along with the corneal power change induced in ortho-k will affect the peripheral retinal defocus. Three studies have investigated the association between the post-ortho-k corneal power change and axial elongation and revealed that a higher amount of corneal power change was associated with better retardation in axial elongation, ${ }^{17-19}$ although one study ${ }^{20}$ reported contradictory findings of no association between corneal power change after short-term and longterm ortho-k lens wear and eye growth. The discrepancies may be due to differences in defining the corneal region and the amount of corneal power change in each study.

The exact mechanisms for optical treatments, including ortho-k, in retardation of axial elongation are still unclear. One popular hypothesis for the effect of slowing axial elongation is that it is due to myopic defocus induced by the optical interventions. ${ }^{16930}$ The effect of retinal defocus on axial elongation using animal models is well documented. ${ }^{31-33}$ There appears to be no significant correlation found between peripheral refraction and myopia progression in humans. ${ }^{34}$ However, myopia progression may be related to the hyperopic defocus and higher-order aberration of the eye..$^{35-38}$

It has been shown that in a contralateral self-controlled study, increasing the compression factor by $1.00 \mathrm{D}$ significantly increases the incidence of ocular higherorder aberrations, primarily spherical aberration. ${ }^{36}$ This increase could therefore improve the myopia control efficacy as axial elongation has been shown to be related to a visually driven mechanism. ${ }^{35} 3738$ Lau et al have shown that higher-order aberration was associated with axial elongation; however, because of the study design, it is unclear whether the effect was due to change in aberration as a result of refractive correction or change in aberration due to normal ageing. ${ }^{39}$ Ocular higher-order aberrations are also measured in the current study and these will be investigated at the end of this study, after 2 years of lens wear.

The initial AL shortening after commencement of ortho-k treatment was significantly higher in the ICF group after 1 month of lens wear, which is in agreement with our previous study. ${ }^{40}$ The anterior segment length has been shown to be unaffected by ortho-k lens wear ${ }^{41}$ and CCT thinning in the ICF group only accounted for around $30 \%$ of the total AL shortening after 1 month of lens wear. The source of the remaining AL shortening was unclear, although it has been suggested to be due to thickening of the choroid. ${ }^{42}$ Retinal defocus in animals and human studies ${ }^{43-47}$ have demonstrated choroidal thickness changes both in response to exposure to spectacle lenses of myopic ${ }^{46}$ or hyperopic defocus, ${ }^{47}$ and in ortho-k-treated eyes. ${ }^{48}$ Again, the choroidal thickness changes and its association between the AL will be investigated at the end of this longitudinal study.

Originally, this current longitudinal study was planned as a randomised clinical trial with three arms; however, because of the popularity of using ortho-k lenses as a treatment in controlling myopia progression in Hong Kong, a partially randomised study design has to be adopted, owing to the high refusal rate in the control group. Popularity of ortho-k for myopia control is likely to be linked to the increase in evidence of the ability of ortho-k to slow myopia progression. ${ }^{9-15}$ An investigation of parents' knowledge of methods for myopia control in Hong Kong revealed that ortho-k was the most widely known method. ${ }^{50}$ Parents are concerned about myopia progression and eager to seek ortho-k treatment for their children, leading to difficulties in recruitment of control subjects in recent studies, making a fully randomised study design impractical.

The spherical power achieved at 1 month for the ICF group was significantly more hyperopic than that of the CCF group. Despite a higher amount of refractive error 
correction, which might imply a higher exertion of pressure on the cornea, there were no significant differences in external ocular health, in terms of corneal staining between the two ortho-k groups. As well as corneal staining, pigmented arc is another commonly observed change at the mid-peripheral cornea after commencement of ortho-k wear. ${ }^{51}{ }^{52}$ A pigmented arc was found in one subject in the CCF group at the 1-month visit and none in the ICF group. Although the refractive change was more rapid in the ICF group, the formation of a pigmented arc was not enhanced in the ICF group, at least, not in the first month of lens wear.

In conclusion, the preliminary results of this study indicated that the increased $1 \mathrm{D}$ in compression factor increased myopia correction without compromising ocular health or the first fit success rate. ICF ortho-k lenses are therefore safe to be used by children and could reduce the time for children to achieve full refractive correction. This study is the first long-term longitudinal study to investigate the differences in efficacy of myopia control and ocular responses between ICF and CCF ortho-k. The results of this study may lead to development of a new ortho-k lens design and provide a better understanding of the mechanism of ortho-k in slowing axial elongation.

Acknowledgements The authors thank Dr Maureen Boost for her advice in the preparation of the manuscript. The authors also thank Mr Bill Yau for his assistance in the transformation of refractive data into Long's formalism.

Contributors All authors designed and planned the study. KW and JKL conducted the experiment. KW analysed the data and wrote the first draft of the paper. All authors interpreted the results, revised the manuscript and approved the final version of the work.

Funding KW and JKL are supported by the Research Residency Scheme provided by The School of Optometry of The Hong Kong Polytechnic University (PolyU). This study is supported by a Collaborative Research Agreement between PolyU and Menicon Co. Ltd., Japan (ZG3Z). Saline and artificial tears are supported by Ophtecs Co., Japan.

Competing interests None declared.

Patient and public involvement Patients and/or the public were not involved in the design, or conduct, or reporting, or dissemination plans of this research.

Patient consent for publication Not required.

Ethics approval All procedures are performed in accordance with the tenets of the Declaration of Helsinki. Ethics clearance has been obtained from the Departmental Research Committee of the School of Optometry at The Hong Kong Polytechnic University (HSEARS20150909002-01).

Provenance and peer review Not commissioned; externally peer reviewed.

Data availability statement No data are available.

Open access This is an open access article distributed in accordance with the Creative Commons Attribution Non Commercial (CC BY-NC 4.0) license, which permits others to distribute, remix, adapt, build upon this work non-commercially, and license their derivative works on different terms, provided the original work is properly cited, appropriate credit is given, any changes made indicated, and the use is non-commercial. See: http://creativecommons.org/licenses/by-nc/4.0/.

ORCID iDs

Kin Wan http://orcid.org/0000-0002-0516-4077

Jason Ki-kit Lau http://orcid.org/0000-0003-0829-6494

\section{REFERENCES}

1 Mountford J. Design variables and fitting philosophies of reverse geometry lenses. In: Mountford J, Ruston D, Dave T, eds. Orthokeratology principles and practice, 2004: 69-107.

2 Maldonado-Codina C, Efron S, Morgan P, et al. Empirical versus trial set fitting systems for accelerated orthokeratology. Eye Contact Lens 2005;31:137-47.

3 Mountford J. Retention and regression of orthokeratology with time. Int Contact Lens Clin 1998;25:59-64.

4 Sorbara L, Fonn D, Simpson T, et al. Reduction of myopia from corneal refractive therapy. Optom Vis Sci 2005;82:512-8.

5 Chan KY, Cheung SW, Cho P. Clinical performance of an orthokeratology lens fitted with the aid of a computer software in Chinese children. Cont Lens Anterior Eye 2012;35:180-4.

6 Fan L, Jun J, Jia Q, et al. Clinical study of orthokeratology in young myopic adolescents. Int Contact Lens Clin 1999;26:113-6.

7 Chan B, Cho P, Cheung SW. Orthokeratology practice in children in a university clinic in Hong Kong. Clin Exp Optom 2008;91:453-60.

8 Chan B, Cho P, Mountford J. The validity of the Jessen formula in overnight orthokeratology: a retrospective study. Ophthalmic Physiol Opt 2008;28:265-8.

9 Charm J, Cho P. High myopia-partial reduction ortho-k: a 2-year randomized study. Optom Vis Sci 2013;90:530-9.

10 Chen C, Cheung SW, Cho P. Myopia control using toric orthokeratology (TO-SEE study). Invest Ophthalmol Vis Sci 2013:54:6510-7.

11 Cho P, Cheung S-W. Retardation of myopia in Orthokeratology (ROMIO) study: a 2-year randomized clinical trial. Invest Ophthalmol Vis Sci 2012;53:7077-85.

12 Cho P, Cheung SW, Edwards M. The longitudinal orthokeratology research in children (LORIC) in Hong Kong: a pilot study on refractive changes and myopic control. Curr Eye Res 2005;30:71-80.

13 Kakita T, Hiraoka T, Oshika T. Influence of overnight orthokeratology on axial elongation in childhood myopia. Invest Ophthalmol Vis Sci 2011;52:2170-4.

14 Santodomingo-Rubido J, Villa-Collar C, Gilmartin B, et al. Myopia control with orthokeratology contact lenses in Spain: refractive and biometric changes. Invest Ophthalmol Vis Sci 2012;53:5060-5.

15 Walline JJ, Jones LA, Sinnott LT. Corneal reshaping and myopia progression. Br J Ophthalmol 2009;93:1181-5.

16 Kang P, Swarbrick H. Peripheral refraction in myopic children wearing orthokeratology and gas-permeable lenses. Optom Vis Sci 2011;88:476-82.

17 Zhong Y, Chen Z, Xue F, et al. Central and peripheral corneal power change in myopic orthokeratology and its relationship with 2-year axial length change. Invest Ophthalmol Vis Sci 2015;56:4514-9.

18 Zhong Y, Chen Z, Xue F, et al. Corneal power change is predictive of myopia progression in orthokeratology. Optom Vis Sci 2014;91:404-11.

19 Lee EJ, Lim DH, Chung T-Y, et al. Association of axial length growth and topographic change in orthokeratology. Eye Contact Lens 2018;44:292-8.

20 Santodomingo-Rubido J, Villa-Collar C, Gilmartin B, et al. Short-term and long-term changes in corneal power are not correlated with axial elongation of the eye induced by orthokeratology in children. Eye Contact Lens 2018;44:260-7.

21 Efron N. Efron grading scales for contact lens complications (Appendix K). In: Efron N, ed. Contact lens practice. 3rd edn. Elsevier Health Sciences, 2016: 453-5.

22 Sakpal TV. Sample size estimation in clinical trial. Perspect Clin Res 2010;1:67

23 Aristodemou P, Sparrow JM, Kaye S. Evaluating refractive outcomes after cataract surgery. Ophthalmology 2019;126:13-18.

24 Wan K, Lau JK-K, Cheung SW, et al. Refractive and corneal responses of young myopic children to short-term orthokeratology treatment with different compression factors. Cont Lens Anterior Eye 2020;43:65-72.

25 Nichols JJ, Marsich MM, Nguyen M, et al. Overnight orthokeratology. Optom Vis Sci 2000;77:252-9.

26 Rah MJ, Jackson JM, Jones LA, et al. Overnight orthokeratology: preliminary results of the Lenses and Overnight Orthokeratology (LOOK) study. Optom Vis Sci 2002;79:598-605.

27 Cho P, Chan B, Cheung SW, et al. Do fenestrations affect the performance of orthokeratology lenses? Optom Vis $\mathrm{Sci}$ 2012;89:401-10.

28 González-Méijome JM, Villa-Collar C, Queirós A, et al. Pilot study on the influence of corneal biomechanical properties over the short term in response to corneal refractive therapy for myopia. Cornea 2008;27:421-6. 
29 Anstice NS, Phillips JR. Effect of dual-focus soft contact lens wear on axial myopia progression in children. Ophthalmology 2011;118:1152-61.

30 Walline JJ, Greiner KL, McVey ME, et al. Multifocal contact lens myopia control. Optom Vis Sci 2013;90:1207-14.

31 Schaeffel F, Glasser A, Howland HC. Accommodation, refractive error and eye growth in chickens. Vision Res 1988;28:639-57.

32 Smith EL, Hung L-F, Huang J. Relative peripheral hyperopic defocus alters central refractive development in infant monkeys. Vision Res 2009;49:2386-92.

33 Smith EL, Kee C-S, Ramamirtham R, et al. Peripheral vision can influence eye growth and refractive development in infant monkeys. Invest Ophthalmol Vis Sci 2005;46:3965-72.

34 Mutti DO, Sinnott LT, Mitchell GL, et al. Relative peripheral refractive error and the risk of onset and progression of myopia in children. Invest Ophthalmol Vis Sci 2011;52:199-205.

35 Zhang N, Yang X-B, Zhang W-Q, et al. Relationship between higherorder aberrations and myopia progression in schoolchildren: a retrospective study. Int $J$ Ophthalmol 2013;6:295.

36 Lau JK, Vincent SJ, Cheung S-W, et al. The influence of orthokeratology compression factor on ocular higher-order aberrations. Clin Exp Optom 2020;103:123-8.

37 Hiraoka T, Kotsuka J, Kakita T, et al. Relationship between higherorder wavefront aberrations and natural progression of myopia in schoolchildren. Sci Rep 2017;7:7876.

38 Lau JK, Vincent SJ, Collins MJ, et al. Ocular higher-order aberrations and axial eye growth in young Hong Kong children. Sci Rep 2018;8:6726.

39 Lau JK, Vincent SJ, Cheung S-W, et al. Higher-order aberrations and axial elongation in myopic children treated with orthokeratology. Invest Ophthalmol Vis Sci 2020;61:22.

40 Lau JK, Wan K, Cheung S-W, et al. Weekly changes in axial length and choroidal thickness in children during and following orthokeratology treatment with different compression factors. Trans/ Vis Sci Technol 2019;8:9
41 Cheung S-W, Cho P. Validity of axial length measurements for monitoring myopic progression in orthokeratology. Invest Ophthalmol Vis Sci 2013;54:1613-5.

42 Swarbrick HA, Alharbi A, Watt K, et al. Myopia control during orthokeratology lens wear in children using a novel study design. Ophthalmology 2015;122:620-30.

43 Zhu X, Park TW, Winawer J, et al. In a matter of minutes, the eye can know which way to grow. Invest Ophthalmol Vis Sci 2005;46:2238-41.

44 Wildsoet C, Wallman J. Choroidal and scleral mechanisms of compensation for spectacle lenses in chicks. Vision Res 1995;35:1175-94.

45 Wallman J, Wildsoet $\mathrm{C}, \mathrm{Xu}$ A, et al. Moving the retina: choroidal modulation of refractive state. Vision Res 1995;35:37-50.

46 Chakraborty R, Read SA, Collins MJ. Monocular myopic defocus and daily changes in axial length and choroidal thickness of human eyes. Exp Eye Res 2012;103:47-54.

47 Chakraborty R, Read SA, Collins MJ. Hyperopic defocus and diurnal changes in human choroid and axial length. Optom Vis Sci 2013;90:1187-98.

48 Chen Z, Xue F, Zhou J, et al. Effects of orthokeratology on choroidal thickness and axial length. Optom Vis Sci 2016;93:1064-71.

49 Li Z, Hu Y, Cui D, et al. Change in subfoveal choroidal thickness secondary to orthokeratology and its cessation: a predictor for the change in axial length. Acta Ophthalmol 2019;97:e454-9.

50 Cheung S-W, Lam C, Cho P. Parents' knowledge and perspective of optical methods for myopia control in children. Optom Vis Sci 2014;91:634-41.

51 Cho P, Chui WS, Cheung SW. Reversibility of corneal pigmented arc associated with orthokeratology. Optom Vis Sci 2003;80:791-5.

52 Cho P, Cheung SW, Mountford J, et al. Incidence of corneal pigmented arc and factors associated with its appearance in orthokeratology. Ophthalmic Physiol Opt 2005;25:478-84. 